\title{
Modeling and SOC estimation of lithium iron phosphate battery considering capacity loss
}

\author{
Junhui $\mathrm{Li}^{1 *}$, Fengjie Gao ${ }^{2}$, Gangui Yan', Tianyang Zhang ${ }^{1}$ and Jianlin $\mathrm{Li}^{3}$
}

\begin{abstract}
Modeling and state of charge (SOC) estimation of Lithium cells are crucial techniques of the lithium battery management system. The modeling is extremely complicated as the operating status of lithium battery is affected by temperature, current, cycle number, discharge depth and other factors. This paper studies the modeling of lithium iron phosphate battery based on the Thevenin's equivalent circuit and a method to identify the open circuit voltage, resistance and capacitance in the model is proposed. To improve the accuracy of the lithium battery model, a capacity estimation algorithm considering the capacity loss during the battery's life cycle. In addition, this paper solves the SOC estimation issue of the lithium battery caused by the uncertain noise using the extended Kalman filtering (EKF) algorithm. A simulation model of actual lithium batteries is designed in Matlab/Simulink and the simulation results verify the accuracy of the model under different operating modes.
\end{abstract}

Keywords: Lithium-iron battery, Battery model, Capacity fading, State of charge estimation

\section{Introduction}

Wind power generation has been developing rapidly in recent years for being pollution-free and sustainable [1-4]. However, wind power curtailment has become a prominent problem due to the constraints imposed by power dispatch and wind power's fluctuation and unpredictability. Energy storage is an effective means to solve the wind power curtailment problem as it can dynamically absorbs and releases energy. It also realizes the temporal transition of power and energy to effectively eliminate wind power curtailment caused by the system's poor peak regulation ability.

Electrochemical energy storage exemplified by lithium battery has been applied in renewable power generation for its high controllability, modularity, energy density and conversion efficiency [5]. Multiple lithium battery energy storage demonstration projects have been conducted throughout China, including Zhangbei County in Zhangjiakou of Hebei Province (14 MW/63WMh lithium phosphate battery system), Baoqing energy storage station in Shenzhen $(4 \mathrm{MW} / 16 \mathrm{MWh}$ lithium iron

\footnotetext{
* Correspondence: lijunhui@neepu.edu.cn

${ }^{1}$ School of Electrical Engineering, Northeast Electrical Power University, Jilin, China

Full list of author information is available at the end of the article
}

phosphate battery system) etc. To promote the development and application of lithium battery technology, the main task is to develop safe, low-cost and long-life lithium ion battery energy storages [6].

Researches on the modeling, control, and capacity allocation of lithium battery energy storage systems have been reported. In terms of energy storage modeling, a battery is composed of positive electrode, negative electrode and electrolyte. Its charge and discharge are electrochemical process and its voltage and current as well as the resistance of the active materials inside are affected by polarization, temperature and other factors [7-10]. The lithium battery will age and lose capacity due to on-going charge and discharge in its life cycle, and therefore, the capacity assessment on lithium battery is necessary and conducive to the adjustment of its operating status in due time. As battery energy storage is generally expensive, it is thus a key issue to establish an effective battery model to analyze the technical and economic characteristics of energy storage system in new energy application.

In [11], a simplified constant power model is adopted which considers capacity limit but the influence of relevant parameters are neglected. Such simplified model is 
incapable of effectively verifying the application results of energy storage. In [12], a Thevenin's equivalent circuit model is used but it results in significant errors due to the negligence of the influence of the state of charge (SOC) on model parameters. The modeling methods in [13-15] present the corresponding numerical relationship between the open circuit voltage, the resistance, the capacitance and the SOC. However, methods for estimating $S O C$ are not included. In [16], a corresponding spatial model based on the equivalent circuit model of lithium iron battery is proposed where the model parameters are estimated using least square method with variable forgetting factors. However, all the abovementioned models fail to consider the capacity loss during the battery's life cycle. In $[17,18]$, the cycle life of high-power lithium iron phosphate battery is studied. Experiment results indicate that battery aging leads to significant impedance amplification and capacity attenuation during the battery's life cycle. Therefore, it is necessary to monitor the battery capacity to avoid damages caused by over charge and discharge.

In this paper, the state equations based on the equivalent circuit model of lithium iron phosphate battery are established. The rest of this paper is organized as follows. Section 2 describes the modeling of lithium iron phosphate battery based on the Thevenin's equivalent circuit. In Section 3, experimental results under constant current and no-load charging and discharging are provided to analyze the resistance and capacitance in the model under different SOC conditions. Capacity loss and available capacity based on different charging and discharging depths are also discussed. And the methods section extended Kalman filtering algorithm (EKF) to estimate the SOC of lithium battery caused by uncertain noise and verify the feasibility of the method. A simulation model of actual lithium batteries is developed using Matlab/Simulink in Section 4 and Section 5. Finally, Section 6 draws the conclusion.

\section{Equivalent circuit of lithium iron phosphate battery}

Lithium iron phosphate battery is a lithium iron secondary battery with lithium iron phosphate as the positive electrode material. It is usually called "rocking chair battery" for its reversible lithium insertion and de-insertion properties. A lithium iron phosphate battery is usually composed of positive electrode, negative electrode, separator and electrolyte, as shown in Fig. 1. The positive electrode is composed of lithium iron phosphate material and the negative electrode is a mixture of solid active materials (LixC6) and carbon granule. The electrochemical reaction occurs on the interface between the active particles of the positive and negative electrodes and electrolyte.
Lithium iron battery is actually a concentration battery whose charge and discharge are realized by the concentration difference of $\mathrm{Li}+$. Reaction on the positive electrode is:

$$
\mathrm{LiFePO}_{4} \underset{\text { disch arge }}{\stackrel{\text { ch arge }}{\rightleftarrows}} \mathrm{Li}_{(i-x)} \mathrm{FePO}_{4}+x \mathrm{Li}^{+}+x e^{-}
$$

and reaction on the negative electrode is:

$$
x L i^{+}+x e^{-}+6 C \underset{\text { disch arge }}{\stackrel{\text { ch arge }}{\rightleftarrows}} L i_{x} C_{6}
$$

The overall equation is give as:

$$
\mathrm{LiFePO}_{4}+6 x \mathrm{C} \underset{\text { disch arge }}{\stackrel{\text { ch arge }}{\rightleftarrows}} \mathrm{Li}_{(1-x)} \mathrm{FePO}_{4}+x \mathrm{LiC}_{6}
$$

Battery energy storage is difficult to be mathematically modeled in detail with conventional physical models as it is an electrochemical reaction process. The accuracy and applicability of the model need to be balanced. Simple models are unable to reflect batteries' characteristics while detailed models may significantly complicate the solution and application of control strategies. The equivalent circuit modeling is adopted for most of existing systems based on the dynamic characteristics and external characteristic performance of the batteries.

The external characteristics based equivalent circuit modeling is a simple and effective way for electrochemical battery modeling. The equivalent circuit model constructs a circuit network with voltage source, capacitance, resistance, inductance and other electrical components to simulate a battery's external transient and steady-state characteristics. It has been applied widely in electrical engineering for its simplicity as the parameters can be accurately identify. It is convenient to integrate multiple elements and is suitable for mathematical analysis.

The Thevenin's equivalent circuit model, which is the most typical battery model at present, is shown in Fig. 2.

As shown, $U_{b}$ is the output voltage of the lithium battery and $U_{o c}$ is the open circuit voltage representing $S O C$ 's nonlinear function. $\mathrm{C}_{\mathrm{use}}$ is the effective/available capacity, and $R_{s}$ is the battery's ohmic resistance. The two RC links represent the electrochemical polarization and concentration difference of polarization during operation, respectively. $\eta$ is the charge and discharge efficiency. The following state equations can be established according to the second order RC equivalent circuit model and Kirchhoff laws: 


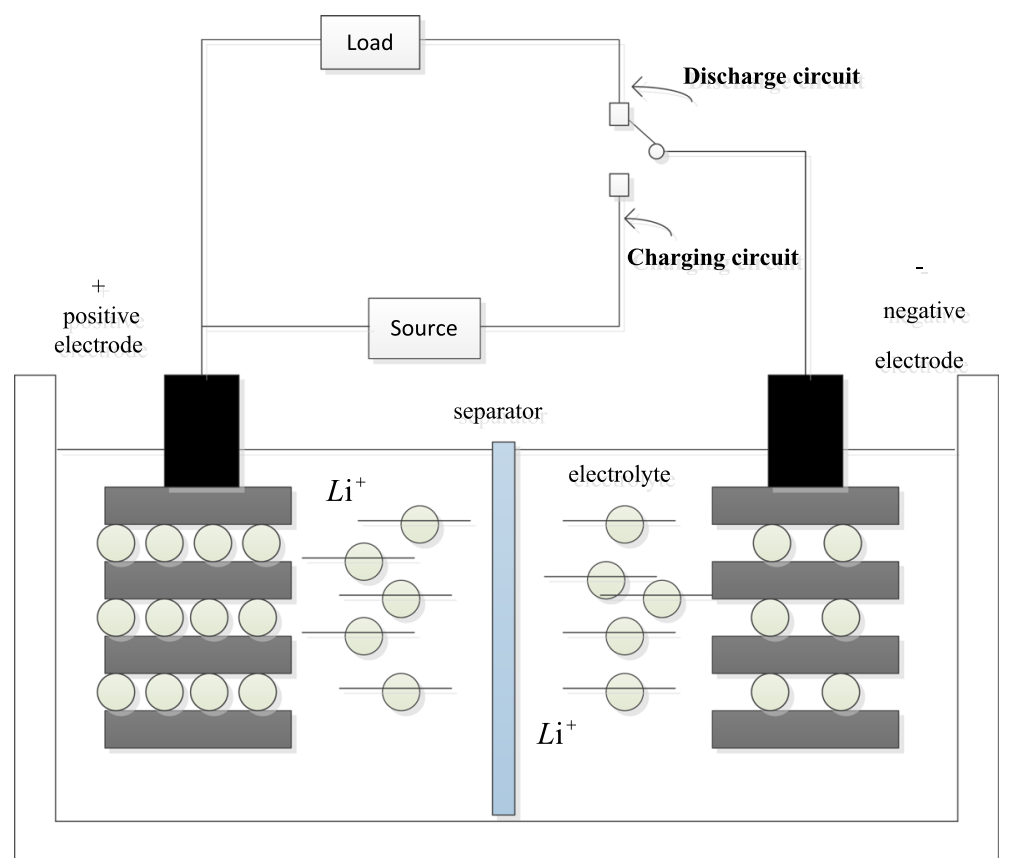

Fig. 1 Schematic diagram of lithium battery

$$
\left\{\begin{array}{l}
U_{b}=U_{o c}-U_{1}-U_{2}-U_{S} \\
\dot{U}_{1}=-\frac{1}{R_{1} C_{1}} U_{1}+\frac{I_{b}}{C_{1}} \\
\dot{U}_{2}=-\frac{1}{R_{2} C_{2}} U_{2}+\frac{I_{b}}{C_{2}} \\
S O C=S O C(0)-\int_{0}^{t} \frac{I_{b} \eta}{C_{u s e}} d t
\end{array}\right.
$$

According to the equivalent circuit model, the left and right circuit networks are coupled and connected by SOC. The state equation indicates that the battery's output voltage is determined by both open circuit voltage and polarization voltage, whereas the polarization voltage is directly related to its corresponding resistance, capacitance and current. The fundamental part of battery modeling is to estimate the available capacity $\left(\mathrm{C}_{\mathrm{use}}\right)$, $S O C$, open circuit voltage, resistance and capacitance of the battery in real time.

\section{Methods}

3.1 Identification of parameters related to battery model Based on Section 2, the parameters of the equivalent circuit model of lithium battery vary with load and external condition as its operating status is affected by discharge depth, cycle number, capacity loss and etc. Therefore, a more reliable model needs be developed by comparing experimental measurements and off-line modeling to establish the relationships between different parameters.

The state of charge (SOC) is the most important influence factor among all the parameters of the resistancecapacitance model. Thus, determining the relationship between impedance parameters and SOC under the battery's standard operation is the primary part of resistance-capacitance modeling. $U_{o c}$ and SOC of lithium battery have a stable relationship under normal operation conditions and is not affected by temperature. Thus, $U_{o c}$ can be considered to be solely determined by

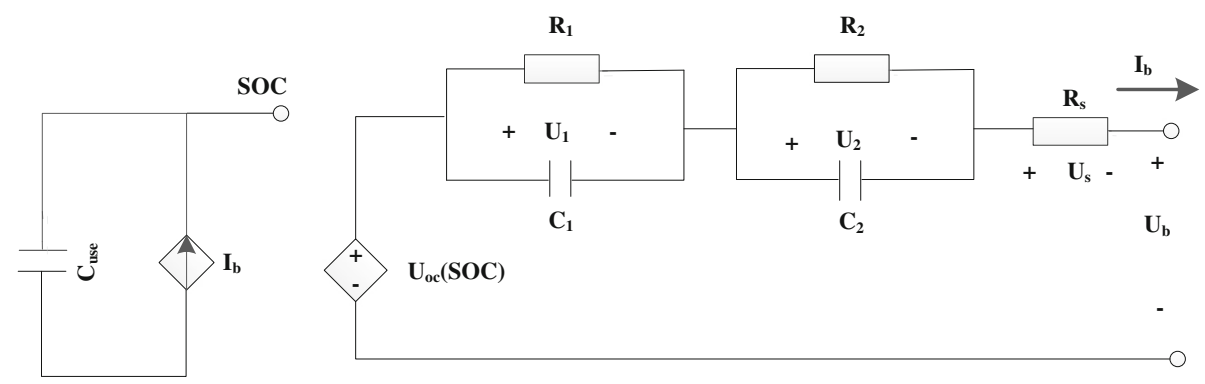

Fig. 2 The external characteristic based equivalent circuit 
SOC and their relationship can be acquired with a fitting function.

The model's resistance and capacitance parameters can be obtained by idling discharge and charge experiments. These experiments are under different SOCs (the initial value and step can be set at 0.2 and 0.02 respectively). The measured voltage and current curves are shown in Fig. 3.

The above waveform indicates that voltage drops sharply in a certain period (OA period) when the battery is discharged while the polarization voltage only changes slightly due to the voltage drop caused by the ohmic resistance $\left(R_{s}\right)$. Thus, it is possible to estimate the ohmic internal resistance inside the battery as the data changes. The terminal voltage then enters a class index variation period ( $\mathrm{AB}$ period) due to the slow drop of polarization voltage $\left(U_{1}, U_{2}\right)$ on the RC circuit, and such period can be considered as a zero-state response period which is described with the following equation:

$$
U_{b}=U_{A}-a \times\left(1-e^{-t / t_{1}}\right)-b \times\left(1-e^{-t / t_{2}}\right)
$$

where $U_{A}$ represents the voltage at A, $a$ and $b$ are the values obtained by curve fitting. To estimate the resistance and capacitance in the RC circuit (6) applies:

$$
\left\{\begin{array}{l}
R_{1}=\frac{a}{I} \\
C_{1}=\frac{\tau_{1}}{R_{1}} \\
R_{2}=\frac{b}{I} \\
C_{2}=\frac{\tau_{2}}{R_{2}}
\end{array}\right.
$$

Equation (7) can be used to estimate the corresponding resistance and capacitance during charge and to obtain the resistance and capacitance values under different SOCs:

$$
U_{b}=U_{D}+a \times\left(1-e^{-t / t_{1}}\right)+b \times\left(1-e^{-t / t_{2}}\right)
$$

where the parameters $a, b, t_{1}$ and $t_{2}$ are the same as in (5).

The $R$ and $C$ values under different states can be acquired by conducting spline interpolation.

\subsection{Assessment of battery's available capacity}

A battery has a limited service life. Because of the continuous charge and discharge during the battery's life cycle, the lithium iron loss and active material attenuation in the lithium iron phosphate battery could cause irreversible capacity loss which directly affects the battery's service life. A real-time capacity assessment on the battery can facilitate the correct recognition of the battery's real-time status and the prediction on the battery's status at certain time point in the future.

The relationship between the lithium battery operation at $\triangle S O C=x$ and the corresponding maximum charge and discharge cycle number $\left.N m\right|_{\triangle S O C=x}$ is fitted based on the experimental data. The fitting function is shown in (8) and is used to calculate the maximum charge and discharge cycle number of lithium battery under a certain charge and discharge circulation depth during its life cycle:

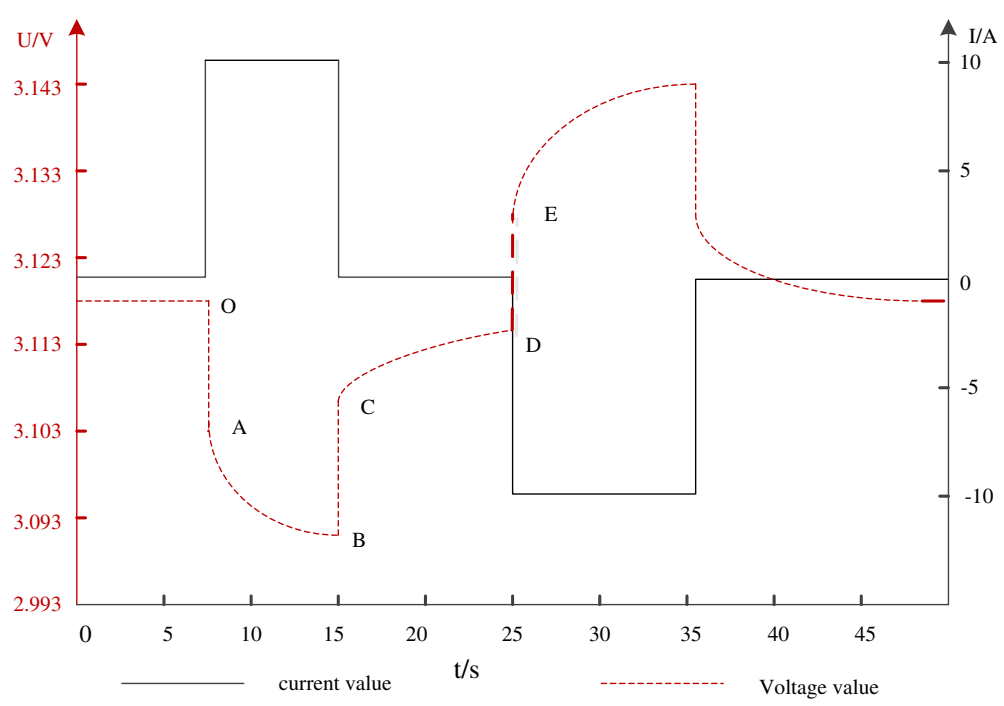

Fig. 3 The experimental curves of discharge and charge for constant current and no-load loading 


$$
\begin{gathered}
\left.N_{m}\right|_{\triangle S O C=x}=640600 x^{8}-2975000 x^{7}+5825000 x^{6} \\
-62800002 x^{5}+40980002 x^{4}-1691000 x^{3} \\
+455900 x^{2}-83820 x+12760
\end{gathered}
$$

Figure 4 shows the maximum charge and discharge cycle numbers when the lithium battery operates at different $\triangle S O C$ s. It can be seen that the battery has different maximum charge and discharge cycles under different $\triangle S O C$ s, with the cycle number being the largest under shallow charge/discharge. The ratio between the cycles under full charge/discharge and under various $\triangle S O C$ s is defined as:

$$
\alpha(x)=\frac{N_{m}(1)}{N_{m}(x)}
$$

where $N_{m}(x)$ and $N_{m}(1)$ are the battery's maximum cycle numbers when the charge and discharge depth equals $x$ $(x \in(0,1))$ and 1 , respectively.

Assuming the battery is charged and discharged for $\mathrm{n}$ times and the charge and discharge depths are $x_{0}, x_{1}, \ldots$ and $x_{n}$ respectively, the battery's equivalent charge and discharge times can be obtained by adding up the equivalent charge and discharge coefficients under different charge and discharge depths as:

$$
N_{m}^{\prime}=\sum_{k=1}^{\mathrm{n}} \alpha\left(x_{k}\right)
$$

The battery's state of health ( $\mathrm{SOH}$ ), also called the state of life which reflects the battery's health status, is defined as the ratio between the nominal capacity and the capacity released by the battery discharging at a certain rate from full charge until the cut-off voltage as:

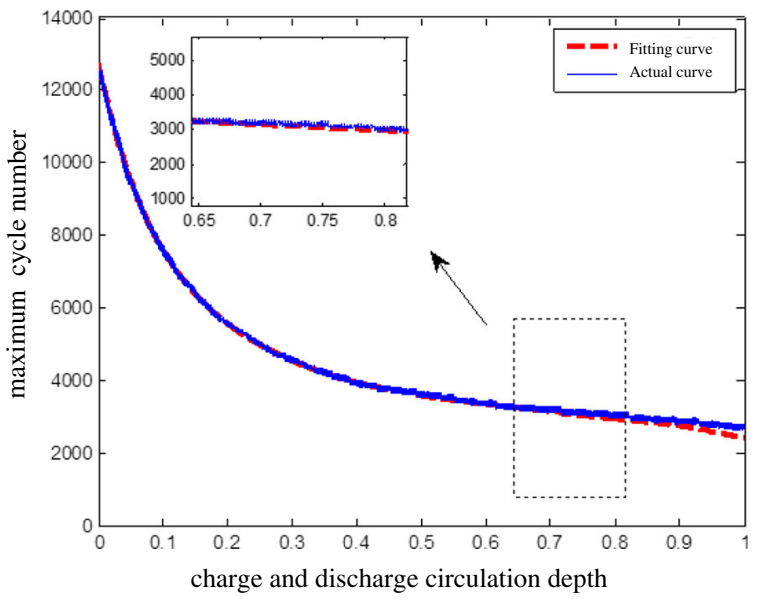

Fig. 4 Relationship between different $5 O C$ and maximum cycle number

$$
S O H=\frac{C_{\text {use }}}{C_{\text {Capicity }}} \times 100 \%
$$

$\mathrm{SOH}$ varies from 0 to $100 \%$, reflecting the battery's health status and indicates the aging degree. A battery would have lost its functions and cannot perform charge and discharge when $\mathrm{SOH}$ is reduced to 20 to $30 \%$ [12].

The available capacity of lithium battery at moment $\mathrm{t}$ can be measured by:

$$
C_{\text {use }}=C_{\text {Capicity }}-\frac{N m^{\prime}}{N m(1)} C_{\text {Capicity }} \times \gamma
$$

where $\gamma$ is a constant and refers to the maximum allowed capacity loss during the battery's normal operation, i.e. the maximum $\mathrm{SOH}$ value. In this paper $\gamma$ is set at 0.3 .

\subsection{State estimation on SOC with EKF algorithm}

According to the previous sections, SOC is an important parameter influencing the safe and reliable operation of lithium battery, and accurate SOC estimation can facilitate the real-time adjustment of control strategy by operators.

The Kalman filtering algorithm is composed of state equation, output equation and the statistical characteristics of system process noise and observation noise. The required states or parameters are calculated according to the system's state equation and output equation. This algorithm can perform optimal minimum variance estimation on SOC and facilitate the prediction and estimation on battery at a certain moment in the future. The conventional Kalman filtering algorithm is a state equation with a linear system while the extended Kalman filtering algorithm (EFK) is required for nonlinear models such as battery. This paper adopts EKF to conduct estimation on the battery's real-time SOC state with the following procedure.

The Kalman filtering state equation and output equation of lithium battery based on its equivalent mathematical model is established. The state equation is given by:

$$
\begin{gathered}
{\left[\begin{array}{c}
\operatorname{SOC}(k+1) \\
u_{1}(k+1) \\
u_{2}(k+1)
\end{array}\right]=\left[\begin{array}{ccc}
1 & 0 & 0 \\
0 & e^{-\frac{\Delta t}{\tau_{1}}} & 0 \\
0 & 0 & e^{-\frac{\Delta t}{\tau_{2}}}
\end{array}\right] \times\left[\begin{array}{c}
\operatorname{SOC}(k) \\
u_{1}(k) \\
u_{2}(k)
\end{array}\right]} \\
+\left[\begin{array}{c}
-\frac{\eta \Delta t}{C_{u s e}} \\
R_{1}\left(1-e^{-\frac{\Delta t}{\tau_{1}}}\right) \\
R_{2}\left(1-e^{-\frac{\Delta t}{\tau}}\right)
\end{array}\right] \times i(k)+w(k)
\end{gathered}
$$

and the output equation is: 


$$
\begin{gathered}
u_{b}(k)=u_{o c}(k)-i(k) \times R_{s}(k) \\
-u_{1}(k)-u_{2}(k)+v(k)
\end{gathered}
$$

For general patterns corresponding to the Kalman filtering state equation, let:

$$
\begin{aligned}
& A_{k}=\left[\begin{array}{ccc}
1 & 0 & 0 \\
0 & e^{\frac{\Delta t}{t_{1}}} & 0 \\
0 & 0 & e^{\frac{\Delta t}{\tau_{2}}}
\end{array}\right] \quad B_{k}=\left[\begin{array}{c}
-\frac{\eta \Delta t}{C} \\
R_{1}\left(1-e^{\frac{\Delta t}{\tau_{1}}}\right) \\
R_{2}\left(1-e^{\frac{\Delta t}{\tau_{2}}}\right)
\end{array}\right] \\
& X_{k}=\left[\begin{array}{c}
S O C(k) \\
U_{1}(k) \\
U_{2}(k)
\end{array}\right]
\end{aligned}
$$

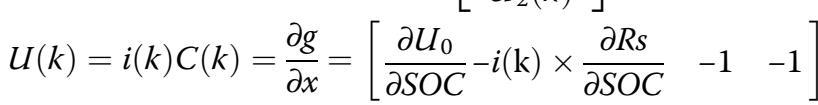

The real-time SOC can be estimated according to the flow chat shown in Fig. 5.

In Fig. 5, $k \mid k-1$ and $k-1 \mid k-1$ refer to the results of the previous state prediction and the optimal results of the previous moment, respectively. $P(k), Q(k)$ and $R(k)$ correspond to the covariances of $X(k), W(k)$ and $V(k)$.

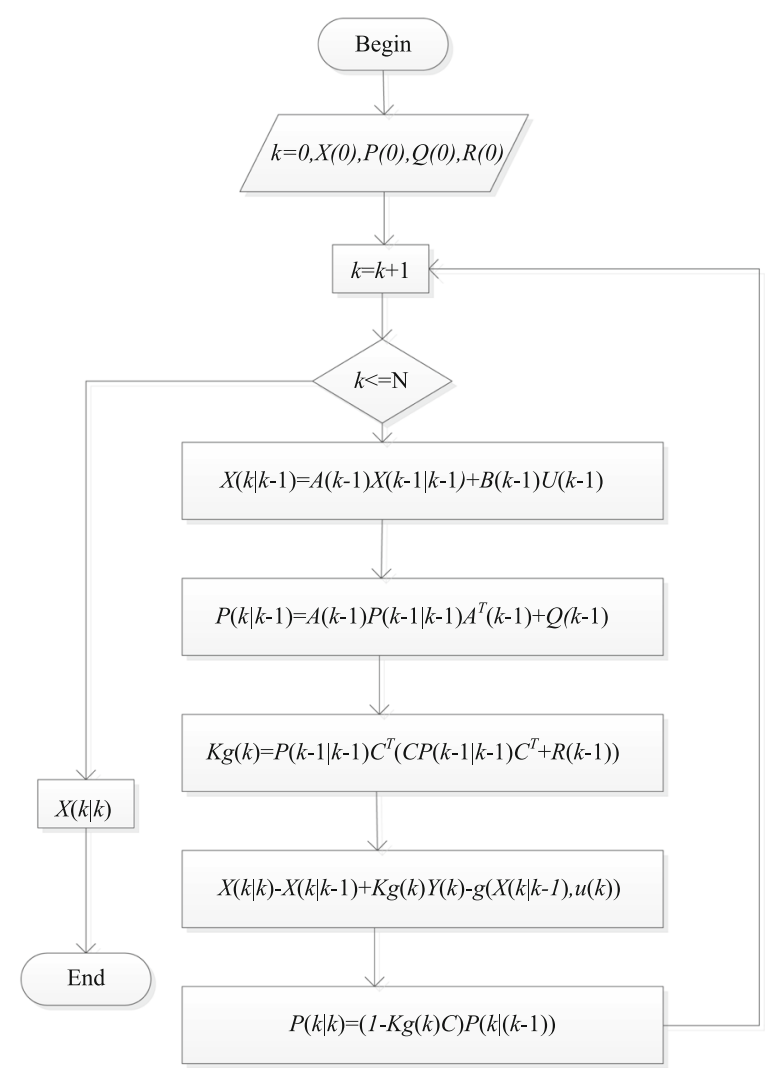

Fig. 5 Flow chart for the Kalman filtering algorithm
The initial values must be selected for the Kalman filtering algorithm, and $X(k)$ contains the three state parameters of $S O C(k), U_{1}(k)$ and $U_{2}(k)$. The $S O C$ at the final moment of the last operation can be selected as the initial value. Since the battery has insignificant effect during the initial operation, the polarization voltage can be considered as 0 . The covariances $Q(k)$ and $R(k)$ are defined as:

$$
\begin{aligned}
& Q(k)=E\left[w(k) \times w(k)^{T}\right] \\
& R(k)=E\left[v(k) \times v(k)^{T}\right]
\end{aligned}
$$

and they are considered as [15]

$$
\begin{gathered}
Q(0)=\left[\begin{array}{ccc}
0.001 & 0 & 0 \\
0 & 0.001 & 0 \\
0 & 0 & 0.001
\end{array}\right] P(0)=\left[\begin{array}{ccc}
0.1 & 0 & 0 \\
0 & 0.01 & 0 \\
0 & 0 & 0.001
\end{array}\right] \\
R(0)=0.001
\end{gathered}
$$

\section{Results}

The data is collected from experiments on domestic lithium iron phosphate batteries with a nominal capacity of $40 \mathrm{AH}$ and a nominal voltage of $3.2 \mathrm{~V}$. The parameters related to the model are identified in combination with the previous sections and the modeling is performed in Matlab/Simulink to compare the output changes between 500 and 1000 circles. Meanwhile, the SOC is estimated with EKF under certain current and voltage for verification.

The obtained relationships between the open circuit voltage and SOC from the measurement and fitted curve are shown in Fig. 6 where an obvious nonlinear relationship can be observed. The fitted curve is obtained using (17) and can accurately estimate the open circuit voltages under different SOCs.

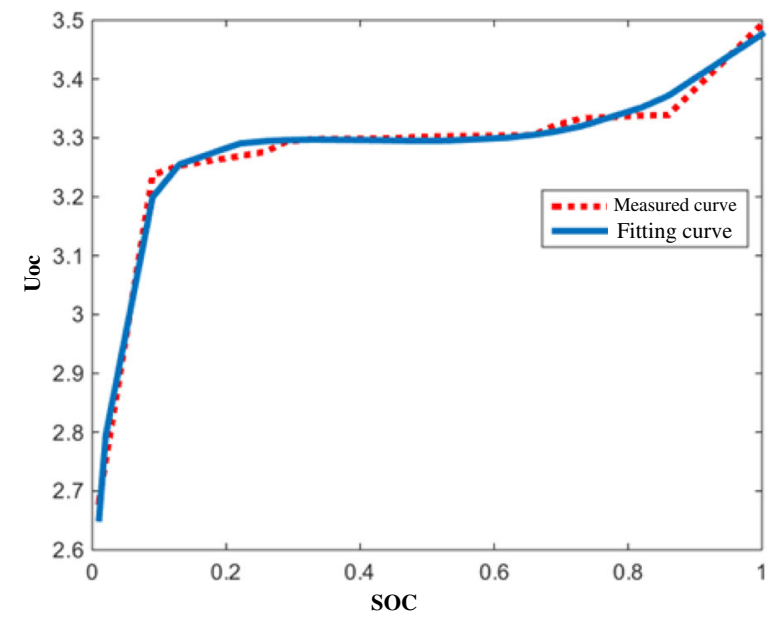

Fig. 6 Relationship between open circuit voltage and SOC 


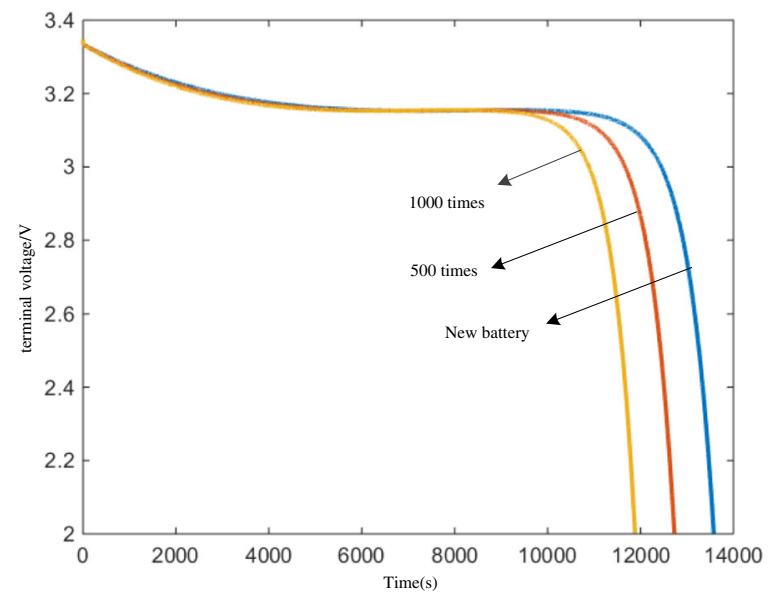

Fig. 7 Discharge curves after different cycles

$$
\begin{gathered}
U_{O C}=-0.7644 e^{-26.6346 \times S O C}+3.2344+0.4834 \times S O C \\
-1.2057 \times S O C^{2}+0.9641 \times S O C^{3}
\end{gathered}
$$

The mathematical model for capacity assessment illustrated in section 4 is used for the discharge experiment on new and used batteries under the same experimental condition. The discharge curves in 500, 1000 and 1500 cycles are shown as Fig. 7. It indicates that, at the same discharge rate, the batteries' voltages displays significant differences towards the end of the discharge period after different cycle numbers. The terminal voltage drops and the discharge cut-off voltage is reached most quickly after 1000 cycles.

Figure 8 further compares the variation of terminal voltage under different SOCs between measured and

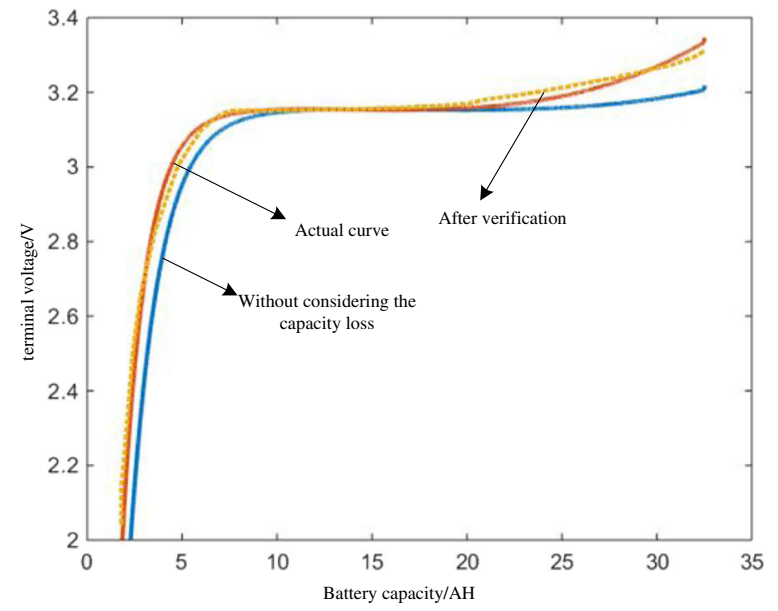

Fig. 8 Variation of measured and estimated terminal voltage with capacity after 1000 cycles

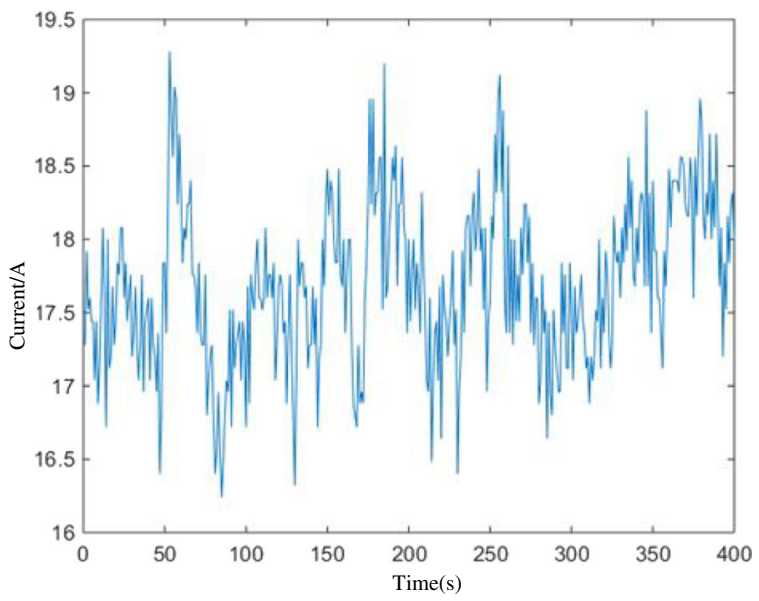

Fig. 9 Variation of partial current with time

estimated ones with and without considering battery capacity loss after 1000 cycles.

It is shown that significant errors could occur in the adjacent period of the SOC estimation without considering capacity loss. It can also be seen that the batteries have significant output voltage variation under different SOCs which poses seriously challenges to the accuracy of the modeling and output voltage estimation.

Table 1 shows that calculation error varies under different SOC intervals, especially under low and high SOCs. Without considering the lithium battery capacity loss, the maximum calculation error is $11.2 \%$ after 1000 cycles, whereas the maximum error is reduced to $3.8 \%$ after the capacity loss is considered in the model. Therefore, the estimation of the current battery capacity is crucial and conducive for improving the battery modeling accuracy and correctly predicting the operation status.

\section{Discussion}

As the battery energy storage system presents "random" charge and discharge characteristics during application, the battery's current may change significantly. In such cases, the conventional Ah counting method can result in significant errors while the extended Kalman filtering algorithm is a better choice. A more accurate SOC can be obtained quickly based on the established model according to the measured current and voltage.

Table 1 Voltage errors between the actual and estimated values with and without considering capacity loss

\begin{tabular}{llll}
\hline SOC & $(0,0.3)$ & $(0.3,0.7)$ & $(0.7,1)$ \\
\hline $\begin{array}{l}\text { Voltage error without considering } \\
\text { capacity loss }\end{array}$ & $0.3-0.4 \mathrm{~V}$ & $0-0.03 \mathrm{~V}$ & $0-0.1 \mathrm{~V}$ \\
$\begin{array}{l}\text { Voltage error considering capacity } \\
\text { loss }\end{array}$ & $0-0.1 \mathrm{~V}$ & $0-0.02 \mathrm{~V}$ & $0-0.02 \mathrm{~V}$ \\
\hline
\end{tabular}




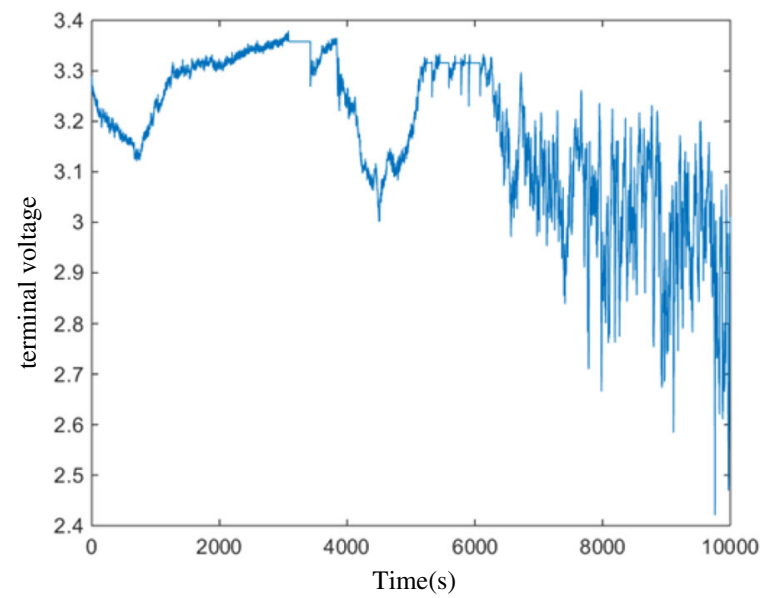

Fig. 10 Variation of battery terminal voltage with time

Figures 9 and 10 show the battery's partial operating condition and the corresponding voltage, respectively.

The changes of SOC calculated by EKF according to the changes of current and voltage is shown in Fig. 11 and is compared to measured values. As shown, the estimation results of SOC using EKF can adequately reflect the actual value and simulate the nonlinear dynamic characteristics of the battery. Therefore, the proposed method can be used for real-time estimation of SOC in energy management system.

\section{Conclusions}

A battery's capacity reduces during the life cycle which affects the estimation of the battery status. This paper carries out a comprehensive study on cycle numbers, discharge depth, current and the capacity reduction during a life cycle. Methods for identifying parameters related to the lithium battery model based on the equivalent circuit are presented, and a mathematical

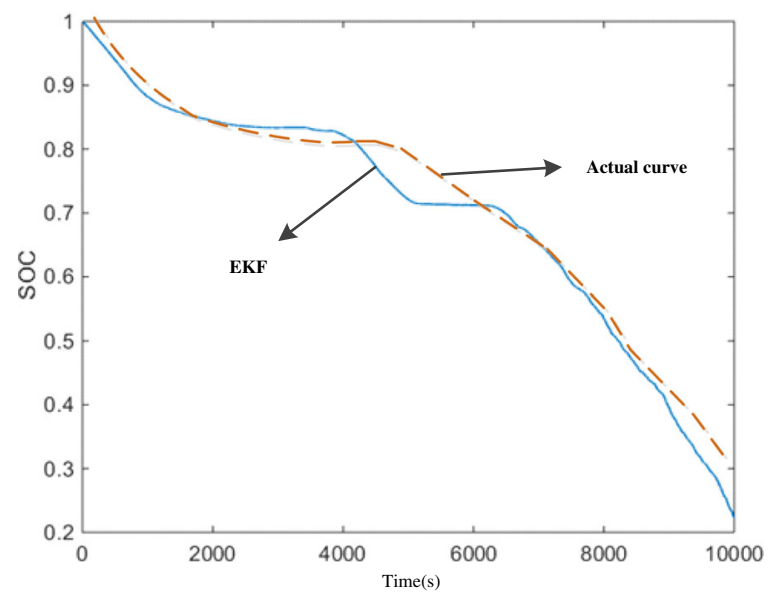

Fig. 11 Variation of SOC with time model for battery capacity estimation is proposed. Method for estimating SOC based on the established mathematical model is presented to facilitate battery energy management and real-time status adjustment. The case studies highlight the followings:

(1)Considering the capacity loss during the battery's life cycle significantly improves the estimation accuracy on the real-time operation status and facilitates the adjustment of related states.

(2) The extended Kalman filtering algorithm for SOC estimation and system discretization can be used for both computer programming and the establishment of energy management system.

\section{Acknowledgements}

This work is supported in part by Open Fund of State Key Laboratory of Operation and Control of Renewable Energy \& Storage Systems (DGB51201700424), Industrial Innovation of Jilin Province Development and Reform Commission (2017C017-2), and Jilin Provincial "13th Five-Year Plan" Science and Technology Project([2016] 88).

\section{Authors' contributions}

$\mathrm{J}$ contributed to the conception of the study and manuscript submission as a corresponding author. FG and GY contributed significantly to analysis and manuscript preparation. TZ revised the manuscript. JL helped perform the study analysis with constructive discussions and senior professional advice. All authors read and approved the final manuscript.

\section{Competing interests}

The authors declare that they have no competing interests.

\section{Author details}

${ }^{1}$ School of Electrical Engineering, Northeast Electrical Power University, Jilin, China. ${ }^{2}$ Dongying Electric Power Supply Company, State Grid Shandong Electric Power Company, Dongying, China. ${ }^{3}$ State Key Laboratory of Operation and Control of Renewable Energy \& Storage Systems, China Electric Power Research Institute, Beijing, China.

Received: 3 June 2017 Accepted: 23 January 2018

Published online: 27 February 2018

\section{References}

1. Sahu, B. K. Hiloidhari, M., \& Baruah, D. C. (2013). Global trend in wind power with special focus on the top five wind power producing countries Renewable \& Sustainable Energy Reviews, 19(1), 348-359.

2. Li, H., Eseye, A. T., Zhang, J., et al. (2017). Optimal energy management for industrial microgrids with high-penetration renewables. Protection \& Control of Modern Power Systems, 2(1), 12

3. Bo, L., Zhijia, H., \& Hao, J. (2016). Wind power status and development trends. Journal of Northeast Electric Power University, 36(02), 7-13.

4. Xiaoming, Y., Shijie, C., \& Jinyu, W. (2013). Prospects analysis of energy storage application in grid integration of large-scale wind power. Automation of Electric Power Systems, 37(1), 14-18.

5. China Industrial Association of Power Sources. (2016). China Energy Storage Industry Report (2016)[R]. Shenzhen: CIBF.

6. Yi, L., Guojun, T., \& Xiaoqun, H. (2017). Optimized battery model based adaptive sigma Kalman filter for state of charge estimation. Transactions of China Electrotechnical Society, 32(2), 108-118.

7. Xikun, C., \& Dong, S. (2016). Research on lithium-ion battery modeling and model parameter identification methods. Proceedings of the CSEE, 36(22), 6254-6261.

8. Chen, Q., Zhao, X., \& Gan, D. (2017). Active-reactive scheduling of active distribution system considering interactive load and battery storage. Protection \& Control of Modern Power Systems, 2(1), 29. 
9. Cuiping, L., Pujia, C., Junhui, L., et al. (2017). Review on reactive voltage control methods for large-scale distributed PV integrated grid. Journal of Northeast Electric Power University, 37(02), 82-88.

10. Xiaoyu, L., Chunbo, Z., Guo, W., et al. (2016). Online parameter estimation of a simplified impedance spectroscopy model based on the fractional joint Kalman filter for life $\mathrm{PO}_{4}$ battery. Transactions of China Electrotechnical Society, 31(24), 141-149.

11. Yang, B., Jingmei, Y., Yingkai, Z., et al. (2017). A real-time rain flow algorithm and its application to state of health modeling for $\mathrm{LiCoO}_{2}$ lithium-ion batteries. Proceedings of the CSEE, 37(12), 3627-3635.

12. Xikun, C., Dong, S., \& Xiaohu, C. (2015). Modeling and state of charge robust estimation for lithium-ion batteries. Transactions of China Electrotechnical Society, 30(15), 141-147.

13. Li, X., Huang, Y., Huang, J., et al. (2014). Modeling and control strategy of battery energy storage system for primary frequency regulation, International Conference on Power System Technology (pp. 543-549). IEEE.

14. Junhui, L., Lian, J., Cuiping, L., et al. (2017). Control strategy designed for converter of super capacitor energy storage system. Journal of Northeast Electric Power University, 37(04), 32-38.

15. Roscher, M. A., Assfalg, J., \& Bohlen, O. S. (2011). Detection of utilizable capacity deterioration in battery systems. IEEE Transactions on Vehicular Technology, 60(1), 98-103.

16. Wei, S., Jiuchun, J., Yanru, Z., et al. (2015). Capacity fading and degradation mechanism of A123 battery. Power System Technology, 39(4), 899-903.

17. Jinlong, Z., Wei, T., Duankai, L., et al. (2017). Rate capacity modeling and state of charge estimation of LiFePO4 battery. Transactions of China Electrotechnical Society, 32(7), 215-222.

18. Zhou, X., Zhang, B., Zhao, H., et al. (2013). State of charge estimation based on improved Li-ion battery model using extended Kalman filter[C], IEEE 8th Conference on Industrial Electronics and Applications (pp. 607-612). IEEE.

\section{Submit your manuscript to a SpringerOpen ${ }^{\circ}$ journal and benefit from:}

- Convenient online submission

- Rigorous peer review

- Open access: articles freely available online

- High visibility within the field

- Retaining the copyright to your article

Submit your next manuscript at $\boldsymbol{s p r i n g e r o p e n . c o m ~}$ 